\title{
ON FUCHSIAN GROUPS WITH THE SAME SET OF AXES
}

\author{
D. D. LONG AND A. W. REID
}

\section{Introduction}

Let $\Gamma$ be a Fuchsian group. We denote by $\operatorname{ax}(\Gamma)$ the set of axes of hyperbolic elements of $\Gamma$. Define Fuchsian groups $\Gamma_{1}$ and $\Gamma_{2}$ to be isoaxial if $\operatorname{ax}\left(\Gamma_{1}\right)=\operatorname{ax}\left(\Gamma_{2}\right)$. The main result in this note is to show (see Section 2 for definitions) the following.

THEOREM 1.1. Let $\Gamma_{1}$ and $\Gamma_{2}$ be isoaxial arithmetic Fuchsian groups. Then $\Gamma_{1}$ and $\Gamma_{2}$ are commensurable.

This result was motivated by the results in [6], where it is shown that if $\Gamma_{1}$ and $\Gamma_{2}$ are finitely generated non-elementary Fuchsian groups having the same nonempty set of simple axes, then $\Gamma_{1}$ and $\Gamma_{2}$ are commensurable. The general question of isoaxial was left open. Theorem 1.1 is therefore a partial answer. Arithmetic Fuchsian groups are a very special subclass of Fuchsian groups (for example, there are at most finitely many conjugacy classes of such groups of a fixed signature), and the general case at present seems much harder to resolve.

The technical result of this paper which implies Theorem 1.1 is Theorem 2.4 (below), proved in Section 4. Denoting the commensurability subgroup by $\operatorname{Comm}(\Gamma)$ and the subgroup of $\operatorname{PGL}(2, \mathbf{R})$ which preserves the set $\operatorname{ax}(\Gamma)$ by $\Sigma(\Gamma)$ (careful definitions are given below), we have the following theorem which describes the commensurability subgroup of an arithmetic Fuchsian group geometrically.

Theorem 2.4. Let $\Gamma$ be an arithmetic Fuchsian group. Then $\operatorname{Comm}(\Gamma)=\Sigma(\Gamma)$.

The problem of 'isoaxial implies commensurable' is like a dual problem to 'isospectral implies commensurable'. It was shown in [8] that this latter statement holds for arithmetic Fuchsian groups. As in the case considered here, the general statement about isospectrality implying commensurability is still open.

\section{Preliminaries}

Here we collect together some arithmetic facts that we shall use, together with a statement of the main technical result required in the proof of Theorem 1.1.

Received 2 August 1996; revised 13 August 1997.

1991 Mathematics Subject Classification 20H10.

The first author was supported by the NSF, and the second by the Royal Society and the NSF.

Bull. London Math. Soc. 30 (1998) 533-538 
2.1. We begin by recalling some notation for quaternion algebras; see [10].

Let $k$ be a field of characteristic $\neq 2$. By a quaternion algebra $A$ over $k$, we mean a 4-dimensional vector space over $k$ such that $A$ is spanned over $k$ by $\{1, i, j, i j\}$, which are subject to the relations $i^{2}=a, j^{2}=b$ and $i j=-j i$, where $a$ and $b$ are non-zero elements of $k$.

Let $A$ be a quaternion algebra over the field $k$, and $x=x_{0}+x_{1} i+x_{2} j+x_{3} i j \in A$. The norm of $x$, denoted $n_{A}(x)$, is given by $x_{0}^{2}-a x_{1}^{2}-b x_{2}^{2}+a b x_{3}^{2}$. $A^{*}$ will denote the invertible elements of $A$, that is, those $x \in A$ with $n_{A}(x) \neq 0$.

If $A$ is a quaternion algebra over a number field $k, v$ a place of $k$, and $k_{v}$ the completion of $k$ at $v$, then $A$ is said to be ramified at $v$ if $A \otimes_{k} k_{v}$ is a division algebra of quaternions. If $v$ is a real place of $k$, then this is equivalent to $A \otimes_{k} k_{v}$ being isomorphic to the Hamiltonian quaternions.

2.2. Throughout, we shall employ the following convention. Given a subgroup $G$ of $\operatorname{GL}(2, \mathbf{R})$, we denote its image in $\operatorname{PGL}(2, \mathbf{R})$ by $\mathrm{PG}$.

A Fuchsian group is a discrete subgroup of $\operatorname{PSL}(2, \mathbf{R})$; however, we sometimes work with Fuchsian groups as subgroups of $\operatorname{SL}(2, \mathbf{R})$.

We now give the definition of an arithmetic Fuchsian group; see [9] or [3], for example.

Let $k$ be a totally real number field, and let $A$ be a quaternion algebra over $k$ which is ramified at all infinite places except one, which we take to be the place associated to the identity embedding of $k$. Via the identity place, we obtain an embedding $\rho: A \hookrightarrow M(2, \mathbf{R})$. Let $\mathcal{O}$ be an order of $A$, and let $\mathcal{O}^{1}$ be the elements of norm 1 in $\mathcal{O}$. Then $\rho\left(\mathcal{O}^{1}\right)$ is a discrete subgroup of $\operatorname{SL}(2, \mathbf{R})$. $\mathrm{P} \rho\left(\mathcal{O}^{1}\right)$ is a finitely generated Fuchsian group of the first kind (so that $\mathbf{H}^{2} / \Gamma$ has finite area).

A Fuchsian group $\Gamma$ in $(\mathrm{P}) \operatorname{SL}(2, \mathbf{R})$ is called arithmetic if $\Gamma$ is commensurable with some group $(\mathrm{P}) \rho\left(\mathcal{O}^{1}\right)$.

2.3. As described in [7], to any non-elementary Fuchsian group $\Gamma$ we can associate a number field (the invariant trace-field), and a quaternion algebra over the invariant trace-field (the invariant quaternion algebra) which is an invariant of the commensurability class of $\Gamma$. Recall that the trace-field of a Fuchsian group $\Gamma$ is simply $\mathbf{Q}(\operatorname{tr}(\gamma): \gamma \in \Gamma)$. Let $\Gamma^{(2)}=\operatorname{gp}\left\{\gamma^{2} \mid \gamma \in \Gamma\right\}$. The invariant trace-field is denoted $k \Gamma$ and is the field $\mathbf{Q}\left(\operatorname{tr}(\gamma): \gamma \in \Gamma^{(2)}\right)$. The algebra

$$
A \Gamma=\left\{\Sigma a_{i} \gamma_{i} \mid a_{i} \in k \Gamma, \gamma_{i} \in \Gamma^{(2)}\right\},
$$

where all sums are finite, is a quaternion algebra over $k \Gamma$ (see, for instance, [1] or [9]). $A \Gamma$ is called the invariant quaternion algebra of $\Gamma$.

Let $\Gamma$ be a finitely generated Fuchsian group of the first kind. Then, in the language discussed above, we have the following characterization of arithmeticity due to Takeuchi [9]. $\Gamma$ is arithmetic if and only if the following conditions hold:

- $k \Gamma$ is a totally real number field;

- traces of elements of $\Gamma$ are algebraic integers;

- $A \Gamma$ is ramified at all infinite places of $k \Gamma$ except one, which is usually taken to be the identity place.

To connect with the definition given in Subsection 2.2, $k \Gamma=k$, and there is some embedding $\rho$ (as above) with $\rho(A)=A \Gamma \subset M(2, \mathbf{R})$. 
2.4. Define the commensurability subgroup of a Fuchsian group $\Gamma$ to be

$$
\operatorname{Comm}(\Gamma)=\left\{\gamma \in \operatorname{PGL}(2, \mathbf{R}) \mid \gamma \Gamma \gamma^{-1} \text { is commensurable with } \Gamma\right\} \text {. }
$$

We have the following result, essentially due to Borel [2]. We give a sketch of the proof, since the first part has relevance in arguments that follow.

THEOREM 2.1. Let $\Gamma$ be an arithmetic Fuchsian group. Then $\operatorname{Comm}(\Gamma)=A \Gamma^{*} / k \Gamma^{*}$.

Proof. Let $A=A \Gamma$ and $k=k \Gamma$. We first show that $\operatorname{Comm}(\Gamma)<A^{*} / k^{*}$. Let $\omega: A^{*} \rightarrow A^{*} / k^{*}$ be the canonical projection (which we also use for the homomorphism $\left.\left(A \otimes_{k} \mathbf{R}\right)^{*} \rightarrow\left(A \otimes_{k} \mathbf{R}\right)^{*} / \mathbf{R}^{*}\right)$.

Let $\gamma \in \operatorname{Comm}(\Gamma)$, and $\gamma_{0} \in \mathrm{GL}(2, \mathbf{R}) \cong\left(A \otimes_{k} \mathbf{R}\right)^{*}$ such that $\omega\left(\gamma_{0}\right)=\gamma$. Since $\gamma \in \operatorname{Comm}(\Gamma)$, it follows that $A \gamma \Gamma \gamma^{-1}=A$, and thus $\gamma_{0}$ induces an automorphism $j_{\gamma_{0}}: A \rightarrow A$ by conjugation. By the Skolem-Noether Theorem (see [10, Chapter 1]), $j_{\gamma_{0}}$ must be inner in the sense that there is $u \in A^{*}$ such that $j_{\gamma_{0}}(a)=u a u^{-1}$ for all $a \in A$. It now follows that $u \gamma_{0}^{-1}$ is central in the quaternion algebra $A \otimes_{k} \mathbf{R}$. Hence $u$ and $\gamma_{0}$ differ by a scalar, so $\omega(u)=\omega\left(\gamma_{0}\right)=\gamma$, and this completes the proof of this direction.

For the reverse inclusion, we simply make the comment that it is not hard to check directly, using an explicit representation of $A$, that $A^{*} / k^{*}<\operatorname{Comm}(\Gamma)$. For one merely shows that choosing $u_{0} \in A^{*}$, one can find a congruence subgroup $\Gamma(I)$ of $\Gamma$ (dependent on the terms in the denominators of the matrix entries of $u_{0}$ ) which is conjugated under $u_{0}$ to a subgroup of finite index in $\Gamma$.

Remark. By the work of Margulis [5], when $\Gamma$ is non-arithmetic, $\operatorname{Comm}(\Gamma)$ is the maximal discrete subgroup of $\operatorname{PGL}(2, \mathbf{R})$ containing $\Gamma$. However, the argument given in the first part of the proof of Theorem 2.1 still shows that $\operatorname{Comm}(\Gamma)<$ $A \Gamma^{*} / k \Gamma^{*}$

2.5. Throughout this subsection, $\Gamma$ will always be a finitely generated Fuchsian group of the first kind. Define $\Sigma(\Gamma)=\{\gamma \in \operatorname{PGL}(2, \mathbf{R}) \mid \gamma(\operatorname{ax}(\Gamma))=\operatorname{ax}(\Gamma)\}$. The following lemma is an elementary observation.

Lemma 2.2. $\Sigma(\Gamma)$ is a subgroup of $\operatorname{PGL}(2, \mathbf{R})$.

If $\Gamma$ is a Fuchsian group and $\gamma \in \Gamma$ is a hyperbolic element, then $\gamma^{n}$ has the same axis as $\gamma$ for all non-zero integers $n$. With this, and the definition of $\operatorname{Comm}(\Gamma)$ given above, we have the following.

\section{Corollary 2.3. $\operatorname{Comm}(\Gamma)<\Sigma(\Gamma)$.}

Proof. If $\Gamma_{1}$ and $\Gamma_{2}$ are commensurable Fuchsian groups, then they contain a finite index subgroup $\Delta$. By the remarks prior to the corollary, we see that $\operatorname{ax}\left(\Gamma_{1}\right)=\operatorname{ax}(\Delta)=\operatorname{ax}\left(\Gamma_{2}\right)$.

Thus, if $x \in \operatorname{Comm}(\Gamma)$, then $x \Gamma x^{-1}$ is commensurable with $\Gamma$, and so $x$ preserves the set $\operatorname{ax}(\Gamma)$. 
The important technical result of this note is the following theorem, to be proved in Section 4. It provides a 'geometric' picture of what $\operatorname{Comm}(\Gamma)$ looks like in the case when $\Gamma$ is arithmetic.

TheORem 2.4. Let $\Gamma$ be an arithmetic Fuchsian group. Then $\operatorname{Comm}(\Gamma)=\Sigma(\Gamma)$.

\section{Jørgensen involutions}

We briefly recall the association of an involution to a pair of non-commuting (hyperbolic) elements of SL(2, C), following Jørgensen [4].

For our purposes, let $g$ and $h$ be a pair of non-commuting hyperbolic elements in $\operatorname{SL}(2, \mathbf{R})$, with fixed points $\left\{a_{g}, r_{g}\right\}$ and $\left\{a_{h}, r_{h}\right\}$, respectively. Since $g$ and $h$ do not commute, they have distinct axes. Define the element $l(g, h)$ of $\mathrm{GL}(2, \mathbf{R})$ by the Lie bracket $g h-h g$. It has the following properties:

- $l(g, h) \cdot g \cdot \imath(g, h)^{-1}=g^{-1}$;

- $l(g, h) \cdot h \cdot l(g, h)^{-1}=h^{-1}$;

- $l(g, h)$ interchanges the fix-points $a_{g}$ and $r_{g}$ (respectively $a_{h}$ and $r_{h}$ ).

The following lemma will be useful.

LEMma 3.1. Let $g_{1}$ and $h_{1}$ be a pair of non-commuting hyperbolic elements, and let $g_{2}$ (respectively $h_{2}$ ) be a hyperbolic element commuting with $g_{1}$ (respectively $h_{1}$ ). Then $l\left(g_{1}, h_{1}\right)$ differs from $\imath\left(g_{2}, h_{2}\right)$ by an element of the centre of $\mathrm{GL}(2, \mathbf{R})$.

Proof. Since $g_{1}$ and $g_{2}$ (respectively $h_{1}$ and $h_{2}$ ) commute, they have the same fixed points. By the properties listed above, we see that the element $\imath\left(g_{1}, h_{1}\right) \cdot \imath\left(g_{2}, h_{2}\right)$ fixes all the fixed points of $g_{1}$ and $h_{1}$-of which there are at least three. By standard properties of Möbius transformations, it follows that $l\left(g_{1}, h_{1}\right) \cdot l\left(g_{2}, h_{2}\right)$ must be a multiple of the identity, and hence in the centre.

\section{Proof of Theorem 2.4}

Before commencing the proof, we make some preliminary comments. Throughout this section, we fix a totally real field $k$ and a quaternion algebra $A$ over $k$ which satisfies the hypothesis in the definition of an arithmetic Fuchsian group given in Subsection 2.1.

We shall make use of the following, which is essentially a consequence of the Skolem-Noether Theorem (see [10, Chapter 1]).

THEOREM 4.1. Let $A$ be a quaternion algebra over a field $k \subset \mathbf{R}$, and let $\rho: A \rightarrow$ $M(2, \mathbf{R})$ be an embedding. Then $\mathrm{P} \rho\left(A^{*}\right)$ is self-normalizing in $\operatorname{PGL}(2, \mathbf{R})$.

Proof. Let $g \in \mathrm{GL}(2, \mathbf{R})$ satisfy $g \rho\left(A^{*}\right) g^{-1}=\rho\left(A^{*}\right)$. Then $g \rho(A) g^{-1}=\rho(A)$, and so by the Skolem-Noether Theorem, this automorphism is inner with respect to an element of $A$. Arguing as in the proof of Theorem 2.1 completes the proof.

We now commence the proof of Theorem 2.4. 
Proof of Theorem 2.4. Without loss of generality, we can assume that $\Gamma=\rho\left(\mathcal{O}^{1}\right)$, where $\mathcal{O}$ is an order of $A$. Let

$$
\mathscr{I}=\{l(g, h) \mid g, h \text { any pair of non-commuting hyperbolic elements } \in \Gamma\},
$$

and let $G(\mathscr{I})$ be the subgroup of $\mathrm{GL}(2, \mathbf{R})$ generated by the elements in $\mathscr{I}$.

We record the following facts about $G(\mathscr{I})$.

Lemma 4.2. $\mathrm{P} G(\mathscr{I})$ is a normal subgroup of $\Sigma(\Gamma)$.

Proof. First, to see that $\mathrm{P} G(\mathscr{I})$ is a subgroup of $\Sigma(\Gamma)$, observe that $l(g, h)=$ $g h-h g \in A \Gamma^{*}$, so that $G(\mathscr{I})<A \Gamma^{*}$. From Corollary 2.3 together with Theorem 2.1, we deduce that $\mathrm{P} A \Gamma^{*}<\Sigma(\Gamma)$. Hence $\mathrm{P} G(\mathscr{I})<\Sigma(\Gamma)$.

Now if $\mathrm{P} \gamma \in \Sigma(\Gamma)$, then for all $l \in \mathscr{I}$ we have $\mathrm{P} \gamma \mathrm{P} l \mathrm{P} \gamma^{-1} \in \mathscr{I}$. To see this, let $\imath=\imath(g, h)$. By definition, $\gamma$ preserves $\operatorname{ax}(\Gamma)$, and so $\gamma$ maps the axes of $g$ and $h$ to the axes of hyperbolic elements $g^{\prime}$ and $h^{\prime}$ in $\Gamma$. Lemma 3.1 implies that the involutions $l\left(\gamma g \gamma^{-1}, \gamma h \gamma^{-1}\right)$ and $i\left(g^{\prime}, h^{\prime}\right)$ differ by a scalar. Hence, in $\operatorname{PGL}(2, \mathbf{R})$, $\mathrm{P} \gamma \mathrm{P} l(g, h) \mathrm{P} \gamma^{-1}=\mathrm{P} l\left(g^{\prime}, h^{\prime}\right)$. The advertised normality now follows from this.

Lemma 4.3. $G(\mathscr{I})$ contains infinitely many non-commuting hyperbolic elements.

Proof. Note that the limit set of $\Sigma(\Gamma)$ coincides with $\mathbf{R} \cup\{\infty\}$, since, as observed above, $\Sigma(\Gamma)$ contains $\mathrm{P} A \Gamma^{*}=\operatorname{Comm}(\Gamma)$. Since $\mathrm{P} G(\mathscr{I})$ is a normal subgroup of $\Sigma(\Gamma)$, they have the same limit set, hence $\mathrm{P} G(\mathscr{I})$ contains a non-elementary subgroup of $\operatorname{PSL}(2, \mathbf{R})$.

The proof of Theorem 2.4 will be completed by showing that $\mathrm{P} A \Gamma^{*}$ is normalized by $\Sigma(\Gamma)$. For then, by Theorem 4.1 , we have $\Sigma(\Gamma)=\mathrm{P} A \Gamma^{*}$ as required.

To establish this claim, we argue as follows.

By Lemma 4.3, $G(\mathscr{I})$ contains a pair of non-commuting elements of infinite order, say $a$ and $b$. Then $B=\{1, a, b, a b\}$ serves as a $k$-basis for $A$. If $\bar{x}=\operatorname{P} x \in \Sigma(\Gamma)$, then by Lemma $4.2, x A \Gamma^{*} x^{-1}$ contains $G(\mathscr{I})$, and hence continues to contain $B$. $x A \Gamma x^{-1}$ is therefore also a quaternion algebra over $k$ with $k$-basis $B$. Hence $x A \Gamma x^{-1}$ and $A \Gamma$ are quaternion algebras over $k$ with the same basis, therefore $A \Gamma=x A \Gamma x^{-1}$, and as conjugation preserves determinant, we deduce that $A \Gamma^{*}=x A \Gamma^{*} x^{-1}$.

\section{Proof of Theorem 1.1 and final remarks}

5.1. We now complete the proof of the main theorem.

THeOREM 1.1. Let $\Gamma_{1}$ and $\Gamma_{2}$ be isoaxial arithmetic Fuchsian groups. Then $\Gamma_{1}$ and $\Gamma_{2}$ are commensurable.

Proof. If $\Gamma_{1}$ and $\Gamma_{2}$ are isoaxial Fuchsian groups, then for any $\gamma \in \Gamma_{2}, \operatorname{ax}\left(\Gamma_{1}\right)=$ $\operatorname{ax}\left(\gamma \Gamma_{1} \gamma^{-1}\right)$, and therefore $\gamma \in \Sigma\left(\Gamma_{1}\right)$.

Since $\Gamma_{1}$ is arithmetic, by Theorem 2.4 we obtain $\Gamma_{2}<\operatorname{Comm}\left(\Gamma_{1}\right)$, and so by Theorem 2.1, $\Gamma_{2}$ is a subgroup of $A \Gamma_{1}^{*} / k \Gamma_{1}^{*}$. Since $\Gamma_{2}$ is also arithmetic, we deduce that $\Gamma_{2}$ is commensurable with $\Gamma_{1}$ by the definition of an arithmetic Fuchsian group.

The natural question that arises from this is whether Theorem 1.1 holds in the non-arithmetic case. At present, we have no reason to believe one way or the other. 
5.2. The methods do extend to arithmetic Kleinian groups with the obvious generalizations of definitions. Thus we simply state (see [3] or [7] for the definition of an arithmetic Kleinian group) the following.

Theorem 5.1. Let $\Gamma_{1}$ and $\Gamma_{2}$ be isoaxial arithmetic Kleinian groups. Then $\Gamma_{1}$ and $\Gamma_{2}$ are commensurable.

ACKNOWLEDGEMENTS. The second author wishes to thank Jim Anderson for useful conversations on this topic.

\section{References}

1. H. Bass, 'Groups of integral representation type', Pacific J. Math. 86 (1980) 15-51.

2. A. Borel, 'Density and maximality of arithmetic subgroups', J. Reine Angew. Math. 224 (1966) 78-89.

3. A. Borel, 'Commensurability classes and volumes of hyperbolic 3-manifolds', Ann. Scuola Norm Sup. Pisa 8 (1981) 1-33.

4. T. JørGensen, 'Compact 3-manifolds of constant negative curvature fibering over the circle', Ann. of Math. (2) 106 (1977) 61-72.

5. G. A. Margulis, Discrete subgroups of semisimple Lie groups, Ergeb. Math. Grenzgeb. (3) (Springer, Berlin, 1991).

6. G. MEss, 'Fuchsian groups with the same simple axes', Preprint (1990)

7. W. D. Neumann and A. W. Reid, 'Arithmetic of hyperbolic 3-manifolds', Topology '90, Proc. Lowdimensional Topology Conference, Ohio State University (de Gruyter, Berlin, 1991) 273-310.

8. A. W. ReID, 'Isospectrality and commensurability of arithmetic hyperbolic 2- and 3-manifolds', Duke Math. J. 65 (1992) 215-228.

9. K. TAKeUCHI, 'A characterization of arithmetic Fuchsian groups', J. Math. Soc. Japan 27 (1975) $600-612$.

10. M-F. VignÉras, Arithmétique des algébras de quaternions, Lecture Notes in Math. 800 (Springer, Berlin, 1980).

Department of Mathematics

University of California

Santa Barbara, CA 93106

USA
Department of Mathematics

University of Texas

Austin, TX 78712

USA 